\title{
Transient dislocation emission from a crack tip
}

\author{
Wei Yang ${ }^{*, 1}$, Jia-Cai Tang ${ }^{1}$, Yi-Shyong Ing ${ }^{2}$, Chien-Ching $\mathrm{Ma}^{2}$
}

Received 20 June 2000

\begin{abstract}
Transient nature of dislocation emission from a crack tip gives a new twist to the study of brittle-to-ductile transition. In a class of materials, only the dislocations traveling at high speed may escape from the crack tip. The nucleation of a fast moving dislocation, however, requires a higher level of activation energy, as supported by many experimental data. The present paper explores this scenario under the restriction that the dislocation moves along the crack extension plane. Fundamental solutions of moving dislocations are derived, and which provide the drag forces on the dislocations and the shielding to the crack tip. Nucleation of a fast moving dislocation is examined under the Peierls-Nabarro theory. Incremental dislocation flux is created continuously from the crack tip, and moves away at a constant speed. At a judgmental time of dislocation emission, the displacement jump relates to the holding force along the crack extension plane by a periodic inter-planar potential, and the singular stress induced by the transient and rate-dependent displacement jump negates the original crack tip singularity. A dynamic overshoot calculation under quasi-steady assumption provides an escape velocity of dislocations. To achieve it, extra activation energy is required for the transient dislocation nucleation and that reduces the dislocation nucleation rate along the crack front. When compared with the rate-insensitive process of cleavage, the transient dislocation emission process allows us to predict the rate dependency of the brittle versus ductile behavior of materials. (C) 2001 Elsevier Science Ltd. All rights reserved.
\end{abstract}

Keywords: A. Dislocations; A. Crack tip; A. Dynamic fracture; Brittle-to-ductile transition; Rate effect

\section{Introduction}

Dislocation emission from a crack tip dictates the brittle-to-ductile transition of materials (Kelly et al., 1967; Rice and Thomson, 1974; Schoeck, 1991; Rice, 1992; Rice and Beltz, 1994). A unique behavior of dislocation free zones in the crack-tip vicinity

\footnotetext{
* Corresponding author. Fax: +86-10-2562768.

E-mail address: yw-dem@tsinghua.edu.cn (W. Yang).

${ }^{1}$ Department of Engineering Mechanics, Tsinghua University, Beijing 100084.

${ }^{2}$ Department of Mechanical Engineering, National Taiwan University, Taipei 10617.
} 
was reported by the TEM observation (Kobayashi and Ohr, 1980, 1981, 1984; Ohr, 1985, 1988). The glide planes of dislocations are usually inclined to the cleavage path (Chiao and Clark, 1989), so that cleavage and dislocation emission are determined by different stress distributions. A solid may cleave after a certain amount of dislocation emission. This "quasi-cleavage" case occurs if the emitting dislocations are confined by finite mobility (Hsia and Argon, 1994), or repelled by pileups in front of obstacles (Hsia et al., 1994), or resisted by lattice friction (Zhu et al., 1996).

Insufficient attention has been paid to the transient nature of the dislocation emission, except several transient analyses by Brock and coworkers (Brock and Jolles, 1987; Brock, 1989; Brock and Wu, 1990a, b) for the anti-plane shear case. The transient effect of dislocation emission gives a new twist to the brittle-to-ductile transition. Atomistic calculations (Yang et al., 1994; Tan and Yang, 1994a, b) indicated that emitted dislocations depart from the crack tip at a velocity of several tenths of the shear wave speed. In a class of materials, Tan and Yang (1995) showed that the dislocation emissions are inherently transient; a dislocation is formed near the crack tip by an atomistic catastrophic process, and the sudden release of energy sets the dislocation in transient motion.

The present work examines this issue from the continuum mechanics aspect. To demonstrate the phenomenon under a clear mathematical analysis, our attention is focused on the case where the dislocation emission plane and the cleavage plane coincide (Rice, 1992). Confined to that circumstance, dislocation emission and cleavage are caused by the same stress distribution along the crack extension line, but different activation mechanisms result in different loading rate responses. A static dislocation emission theory predicts a strict brittle-or-ductile alternative: the crack either extends in a form of cleavage or arrests by dislocation emission, regardless the loading rates. On the other hand, rate effect on the brittle versus ductile transition has been emphasized by many experimental investigations (e.g., Nitzsche and Hsia (1994) for silicon; Kim and Roberts (1994) for sapphire; Booth and Roberts (1997) for TiAl single crystals; and Ebrahimi and Shrivastava (1998) for NiAl single crystals). The paper by Hirsch and Roberts (1996) summarized many testing data for Si that correlated the inverse of the brittle-to-ductile transition temperature as a linearly decreasing function of the logarithmic stress intensity factor rate. The transient nature of dislocation emission leads to a scenario in a class of materials where an overshoot is essential for a nucleated dislocation to escape from the crack tip. Accordingly, an escape speed exists for emission of a dislocation. Only those dislocations traveling above the escape speed may leave the crack tip, and the slower ones are trapped. The nucleation of a fast moving dislocation, however, requires a higher level of activation energy, rendering the loading rate dependency of the brittle to ductile transition. This situation is explored under the following assumptions:

(1) Only a plane strain or an anti-plane shear configuration is considered. The crack tip region where dislocations nucleate and escape is small comparing to the other lengths of relevance. Furthermore, the time scale for transient dislocation emission is small compared to the time scale of remote load variation. By these assumptions, the geometry under investigation reduces to a semi-infinite crack in an otherwise infinite medium, and the loading is prescribed by the remote stress intensity factors. The 
emission of a climb dislocation, an edge dislocation, or a screw dislocation is driven by a sustained mode I, II, or III field.

(2) The emission process of a dislocation is described by two stages: nucleation and escape. In the nucleation stage, the Peierls-Nabarro model is used for an incipient dislocation, as in the static counterparts by Argon (1987), Schoeck (1991, 1994, 1996), Beltz and Rice (1991, 1992), Rice (1992), Rice et al. (1992), and Rice and Beltz (1994). In the escape stage, we describe a fully developed dislocation by a Volterra dislocation.

The transient analyses for both stages rely on the fundamental solutions of moving dislocations presented in the next section. These solutions provide the forces on, and the crack tip shielding by, a climb, an edge, or a screw dislocation that emits from the crack tip at a constant speed. The nucleation of a fast moving dislocation is examined in Section 3 under the Peierls-Nabarro theory, generalized from its counterpart in the static case. Incremental dislocation flux is emitted continuously from the crack tip, and moves away at an assigned speed. At a judgmental instant of dislocation emission, the continuous displacement jump along the crack extension line relates to the local traction by a periodic inter-planar potential, and the singular stress induced by this transient and rate-dependent dislocation strip negates the original crack tip singularity. These requirements allow us to determine the dislocation emitting flux from the crack tip, which serves to evaluate the activation energy for a moving dislocation. A dynamic overshoot calculation under quasi-steady approximation is conducted in Section 4. The escape velocity of dislocations is obtained by integrating a nonlinear first-order differential equation. To achieve this escape velocity, extra activation energy is required for the transient dislocation nucleation, and that consequently reduces the dislocation nucleation rate along the crack front. When compared with the rate-insensitive process of cleavage, the velocity related dislocation emission process allows us to predict the rate dependency of the brittle versus ductile behavior of materials.

\section{Fundamental solutions for moving dislocations}

Consider the plane strain or anti-plane shear deformation of a linear isotropic elastic solid. The elasticity properties are assigned by a shear modulus $\mu$ and a Poisson's ratio $v$. The in-plane coordinates are denoted by $x_{1}$ and $x_{2}$. The geometry for dislocation emission concerns a semi-infinite crack lying along the negative $x_{1}$-axis in an otherwise infinite plane. The body is at rest before a time instant $t_{0}$. At $t=t_{0}$, a dislocation suddenly emerges at the crack tip, and propagates rightward along the crack extension line at a constant velocity $v$. This section analyzes the excitation by emitting such a dislocation, termed the fundamental solution of a moving dislocation. The conventional elastodynamic governing equations are adopted, e.g., see Freund (1990, Section 1.2). The crack faces and the remote boundary are traction free at all time. The process of dislocation emission is visualized as follows. Suppose that a pair of dislocations of opposite Burgers vectors $\pm b_{i}$ sit at the crack tip before $t=t_{0}$, so they exactly cancel each other. Quantities $b_{1}, b_{2}$ or $b_{3}$ correspond to the edge, climb or screw component of the dislocation. Since $t=t_{0}$, the dislocation of Burgers vector $+b_{i}$ moves out at 
a velocity $v$ and the dislocation of Burgers vector $-b_{i}$ remains at the crack tip. A displacement discontinuity of $b_{i}$ is created from the crack tip to the point $x_{1}=\xi$ along the crack extension line

$$
u_{i}\left(x_{1}, 0^{ \pm}, t\right)= \pm \frac{b_{i}}{2} H\left(\xi-x_{1}\right) H\left(x_{1}\right) H\left(t-t_{0}\right),
$$

where $H(\bullet)$ denotes the Heaviside step function, and $\xi=v\left(t-t_{0}\right)$ abbreviates the location of the dislocation.

Freund (1974, 1990, Section 3.2.2) obtained a particular version of the fundamental solution for the transient emission of a climb dislocation. Adopting the same methodology of Laplace transform and Wiener-Hopf technique, we derive an elastodynamic semi-infinite crack solution under the excitation of Eq. (1). The traction along the crack extension line assumes the following simple form (no summation for repeated indices unless explicitly indicated):

$$
\sigma_{2 i}\left(x_{1}, 0, t\right)=-\frac{\mu_{i} b_{i}}{2 \pi\left(\xi-x_{1}\right)} \sqrt{\frac{\xi}{x_{1}}} F_{i}^{+}\left(\frac{1}{v}\right) F_{i}^{-}\left(\frac{t-t_{0}}{x_{1}}\right) H\left[c_{i}\left(t-t_{0}\right)-x_{1}\right] .
$$

In Eq. (2), the elastic constants and the wave speeds generated by different displacement discontinuities are $\mu_{1}=\mu_{2}=\mu /(1-v), \mu_{3}=\mu, c_{2}=c_{\mathrm{L}}, c_{1}=c_{3}=c_{\mathrm{T}}$, with $c_{\mathrm{L}}$ and $c_{\mathrm{T}}$ being the longitudinal and transverse wave speeds. The dimensionless $F$-functions in Eq. (2) are given by

$$
F_{1,2}^{ \pm}(\zeta) \frac{\sqrt{1 \pm 1 / c_{\mathrm{R}} \zeta}}{\sqrt{1 \pm 1 / c_{\mathrm{T}, \mathrm{L}} \zeta}} S_{ \pm}(\zeta), \quad F_{3}^{ \pm}(\zeta)=\sqrt{1 \pm \frac{1}{c_{\mathrm{T}} \zeta}}
$$

where $c_{\mathrm{R}}$ is the Rayleigh wave speed. The functions $S_{ \pm}(\zeta)$ come from the standard process of Wiener-Hopf factorization of the Rayleigh wave function, given by

$$
\log S_{ \pm}(\zeta)=-\frac{1}{\pi} \int_{1 / c_{\mathrm{L}}}^{1 / c_{\mathrm{T}}} \tan ^{-1}\left[\frac{4 z^{2} \sqrt{z^{2}-c_{\mathrm{L}}^{-2}} \sqrt{c_{\mathrm{T}}^{-2}-z^{2}}}{\left(c_{\mathrm{T}}^{-2}-2 z^{2}\right)^{2}}\right] \frac{\mathrm{d} z}{z \pm \zeta} .
$$

As $\zeta \rightarrow \infty$, both $S_{ \pm}(\zeta)$ and $F_{i}^{ \pm}(\zeta)$ asymptotically approach unity.

The denominator in the traction expression (2) indicates an inverse singularity in the vicinity of the moving dislocation. The leading term of the singularity stress is skew-symmetric with respect to the traveling dislocation core, and has no contribution to the force on that dislocation, see Hirth and Lothe (1982, Sections 3.2, 3.4). Excluding this leading term, and taking the limit of $x_{1} \rightarrow \xi$ for the remaining part, one obtains the following expression for the drag stress on dislocation:

$$
\tau_{i}=-\frac{\mu_{i} b_{i}}{2 \pi \xi} F_{i}^{+}\left(\frac{1}{v}\right)\left[\frac{1}{2} F_{i}^{-}\left(\frac{1}{v}\right)+\frac{1}{v} \dot{F}_{i}^{-}\left(\frac{1}{v}\right)\right] .
$$

A super-imposed dot on a function denotes the derivative with respect to its argument. For the case of a moving screw dislocation, the force on it has a simplified expression

$$
\tau_{3}=-\frac{\mu b_{3}}{4 \pi \xi} \sqrt{\frac{c_{\mathrm{T}}+v}{c_{\mathrm{T}}-v}} .
$$




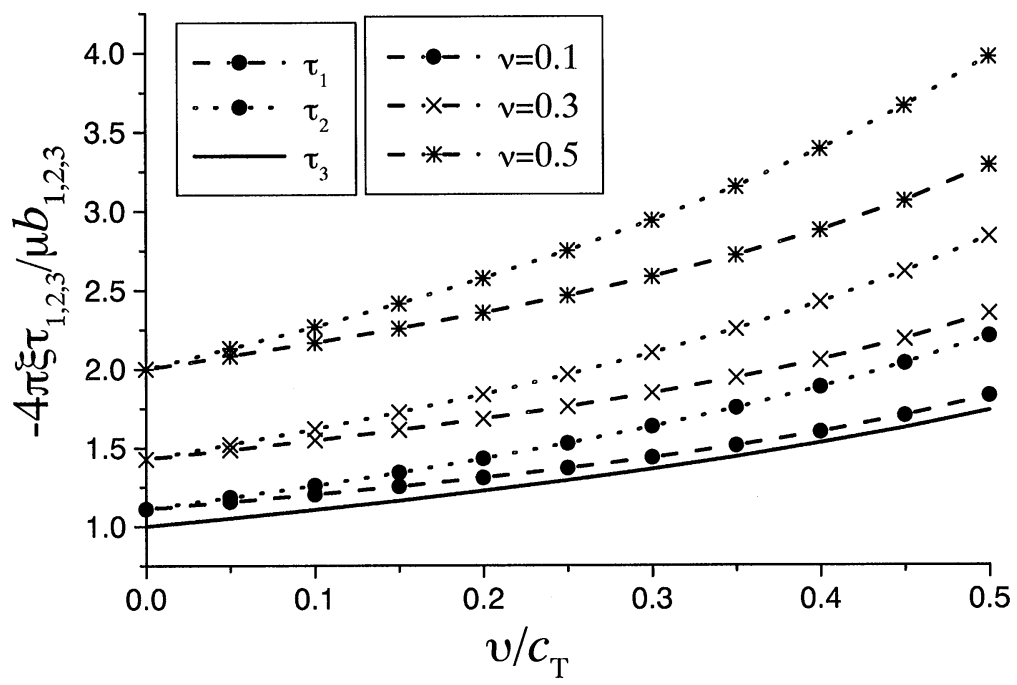

Fig. 1. Variation of the dimensionless dislocation force factor $-4 \pi \tau_{i} \xi /\left(\mu b_{i}\right)$ with respect to the normalized dislocation speed $v / c_{\mathrm{T}}$ for representative Poisson's ratios of $v=0.1,0.3$ and 0.5 .

As can be confirmed by the rigorous analysis by Brock and $\mathrm{Wu}$ (1990a), formula (6) constitutes an exact expression even for the case that dislocation moves at non-uniform velocity. For the dislocation emission under in-plane deformation, formula (5) is specified to

$$
\begin{aligned}
\tau_{1,2}= & -\frac{\mu b_{1,2}}{4 \pi(1-v) \xi} \frac{v^{-1}+c_{\mathrm{R}}^{-1}}{\sqrt{v^{-2}-c_{\mathrm{T}, \mathrm{L}}^{-2}}} S_{+}\left(\frac{1}{v}\right) \\
& \times\left\{\frac{v^{-1}+c_{\mathrm{R}}^{-1}-2 c_{\mathrm{T}, \mathrm{L}}^{-1}}{v^{-1}-c_{\mathrm{T}, \mathrm{L}}^{-1}} S_{-}\left(\frac{1}{v}\right)+2\left(v^{-1}-c_{\mathrm{R}}^{-1}\right) \dot{S}_{-}\left(\frac{1}{v}\right)\right\} .
\end{aligned}
$$

We plot in Fig. 1 the variation of $-\tau_{i}$, normalized by $\mu_{i} b_{i} /(4 \pi \xi)$, with respect to the dislocation speed, normalized by the shear wave speed, for representative Poisson's ratios $v=0.1,0.3$ and 0.5 . It is observed that the drag stress on the dislocation intensifies as the dislocation moves faster. The drag stress on a climb dislocation possesses the strongest velocity dependence, followed by that of an edge dislocation and then a screw dislocation. For the cases of climb and edge dislocations moving in a solid of fixed shear modulus, the higher the Poisson's ratio, the larger the drag stresses.

The fundamental solutions (2) quantify the crack tip shielding for various types of moving dislocations. The shielding stress intensity factors are

$$
K_{\mathrm{I}, \mathrm{II}}^{\text {shield }}=-\frac{\mu b_{2,1}}{(1-v) \sqrt{2 \pi \xi}} \frac{1+v / c_{\mathrm{R}}}{\sqrt{1+v / c_{\mathrm{L}, \mathrm{T}}}} S_{+}\left(\frac{1}{v}\right), \quad K_{\mathrm{III}}^{\text {shield }}=-\frac{\mu b_{3}}{\sqrt{2 \pi \xi}} \sqrt{1+\frac{v}{c_{\mathrm{T}}}} .
$$


The Roman subscripts I, II or III denote the opening, sliding or tearing mode of fracture. For all fracture modes, the strength of the shielding stress intensity factor increases as the corresponding dislocation speed increases.

\section{Transient dislocation nucleation}

This section deals with the transient dislocation nucleation from a crack tip. Advances in the static theory of dislocation nucleation highlight the use of Peierls-Nabarro model, as addressed by Argon (1987), Schoeck (1991, 1994, 1996), Beltz and Rice (1991, 1992), Rice (1992), Rice et al. (1992), Rice and Beltz (1994), and Wang et al. (1996); see also the references therein. Under the Peierls-Nabarro model, the gradual build-up of the dislocation in front of the crack tip is formulated by the emission of a density of infinitesimal dislocations out of the crack tip, and the physical trace of such a gradual dislocation emission was observed recently in nanoscopic resolution near an atomistically sharp crack in single crystal silicon (Xing et al., 2000). The Peierls framework of dislocation emission from a crack tip eliminates the uncertain parameter of core radius, and provides a dislocation core structure from the periodic lattice viewpoint. A counterpart for the transient nucleation is attempted here by exploiting the fundamental solutions in the previous section.

The remote loading is applied by a stress intensity factor $K_{\mathrm{M}}$. Throughout this work, the Latin indices $i=1,2,3$ (for stress and displacement components) correspond to the Roman indices $\mathrm{M}=$ II, I, III (for fracture modes). The loading rate of the stress intensity factor is relatively slow so that an equilibrium stress field is achieved before the emission of dislocation at $t=0$. Before the emergence of a fully developed dislocation (whose Burgers vector has an amplitude $b$ ), a dislocation flux of incremental Burgers vectors is continuously generated at the crack tip. The incremental dislocations travel along the crack extension line at a prescribed speed $v$, so that a moving PeierlsNabarro dislocation dynamically takes shape. To quantify the above ideas, let us denote the emission flux of incremental dislocations from the crack tip by $\left(b / t_{\mathrm{cr}}\right) B_{i}\left(1-t / t_{\mathrm{cr}}\right)$, with $B_{i}(s)(i=1,2,3)$ being a dimensionless crack tip dislocation flux function, and $t_{\mathrm{cr}}$ a critical time to be defined later. The process variable $s$ counts from the critical time $(s=0)$ back to the initial instant of dislocation nucleation $(s=1)$. The displacement jump along the crack extension line at a time $t>0$ is given by

$$
u_{i}\left(x_{1}, 0^{+}, t\right)-u_{i}\left(x_{1}, 0^{-}, t\right) \equiv \delta_{i}\left(x_{1}, t\right)=b \int_{1+\eta-t / t_{\mathrm{cr}}}^{1} B_{i}(s) \mathrm{d} s .
$$

Henceforth, we label the slip length of the incipient dislocation at the critical time as $l_{\mathrm{cr}}=v t_{\mathrm{cr}}$, and define a dimensionless variable $\eta$ as $x_{1} / l_{\mathrm{cr}}$. The value $\eta=0$ denotes the crack tip and the value $\eta=1$ locates the leading edge of the incipient dislocation at the critical moment.

Generated by the emitting dislocation flux, a transient stress adds to the previously equilibrium stress field. The fundamental solutions presented in the previous section enable us to write the traction along the crack extension line by the following 
superposition integral for any $x_{1}$ less than $c_{i} t$ :

$$
\begin{aligned}
\sigma_{2 i}\left(x_{1}, 0, t\right)= & \frac{1}{\sqrt{2 \pi x_{1}}}\left\{K_{\mathrm{M}}-\frac{\mu_{i} b}{\sqrt{2 \pi l_{\mathrm{cr}}}} F_{i}^{+}\left(\frac{1}{v}\right)\right. \\
& \left.\times \int_{1+\eta_{i}-t / t_{\mathrm{cr}}}^{1} F_{i}^{-}\left(\frac{s-1+t / t_{\mathrm{cr}}}{v \eta}\right) \frac{\sqrt{s-1+\left(t / t_{\mathrm{cr}}\right)} B_{i}(s)}{s-\eta-1+t / t_{\mathrm{cr}}} \mathrm{d} s\right\},
\end{aligned}
$$

where $\eta_{i}=\left(v / c_{i}\right) \eta$. The square root singularity near the crack is indicated explicitly in Eq. (10). Due to the transient formation of the incipient dislocation, the stress intensity factor at the crack tip is shielded, and reduced to

$$
K_{\mathrm{M}}^{\mathrm{tip}}(t)=K_{\mathrm{M}}-\frac{\mu_{i} b}{\sqrt{2 \pi l_{\mathrm{cr}}}} F_{i}^{+}\left(\frac{1}{v}\right) \int_{1-t / t_{\mathrm{cr}}}^{1} \frac{B_{i}(s)}{\sqrt{s-1+t / t_{\mathrm{cr}}}} \mathrm{d} s .
$$

At the critical time $t_{\mathrm{cr}}$, the incipient transient dislocation is ready to transform into a fully developed dislocation. Two conditions are imposed at that moment. First, the transient stress field due to the dislocation nucleation should completely shield the crack tip, rendering the crack tip stress intensity vanishes at $t=t_{\mathrm{cr}}$,

$$
K_{\mathrm{M}}=\frac{\mu_{i} b}{\sqrt{2 \pi l_{\mathrm{cr}}}} F_{i}^{+}\left(\frac{1}{v}\right) \int_{0}^{1} \frac{B_{i}(s)}{\sqrt{s}} \mathrm{~d} s .
$$

The second condition states that the traction given in Eq. (10) should relate to the displacement jump given in Eq. (9) by an inter-planar lattice relation

$$
\sigma_{2 i}\left(x_{1}, 0, t_{\mathrm{cr}}\right)=\mu_{i} \gamma\left[\delta_{i}\left(x_{1}, t_{\mathrm{cr}}\right) / b\right]=\frac{\partial \Phi}{\partial \delta_{i}},
$$

where $\gamma$ is a periodic function that has to be inferred by a solid-state physics calculation for a given material. The quantity $\Phi$ in Eq. (13) is termed inter-planar energy. Rice (1992), Rice and Beltz (1994), among many others, adopted a Frenkel sinusoidal form in the cases of gliding dislocations. As a departure from the quasi-static analysis, a dimensionless factor $c(v)$ is introduced to scale the rate effect:

$$
\tau=c(v) \frac{\pi \gamma_{\mathrm{us}}}{b} \sin \left[\frac{2 \pi}{b}\left(\delta+\frac{\tau}{\mu}\right)\right],
$$

where $\tau$ and $\delta$ may refer either mode II (edge dislocation) or mode III (screw dislocation) case. In Eq. (14), $\gamma_{\text {us }}$ is the unstable stacking energy, and $\Delta=\delta+\tau / \mu$ the relative atomic displacement undergone by atoms on the opposite sides of the gliding plane. Relation (14) highlights a dimensionless variable $\gamma_{\mathrm{us}} / \mu_{i} b$ characterizing the lattice resistance to dislocation nucleation. The estimate of $\gamma_{\mathrm{us}} / \mu_{i} b$ (see Rice, 1992) indicates that it falls within the range of $0.03-0.1$ for many materials. The calculations in the sequel are conducted at a representative value of $\gamma_{\mathrm{us}} / \mu b=1 / 2 \pi^{2} \approx 0.050675$ (see Wang, 1995).

Transient dislocation emission, on the one hand, has to shield the crack tip that was previously presided under a stress intensity factor $K_{\mathrm{M}}$; on the other hand, the dislocation emission has to conquer the rate-dependent inter-planar resistance $\tau$ in 
Eq. (14). The former is given in Eq. (12) and has a velocity dependence of $F_{i}^{+}(1 / v)$, while the latter is given in Eq. (14) and has a velocity dependence of $c(v)$. At the critical time $t_{\mathrm{cr}}$, the velocity dependences of the driving force $K_{\mathrm{M}}$ and the resistance $\tau$ should be consistent, leading to

$$
c(v)=F_{i}^{+}\left(\frac{1}{v}\right) \text {. }
$$

Also at $t=t_{\mathrm{cr}}$, the expression (9) reduces to

$$
\delta_{i}\left(x_{1}, t_{\mathrm{cr}}\right)=b \int_{\eta}^{1} B_{i}(s) \mathrm{d} s .
$$

Consequently, the dislocation emission flux $B_{i}(s)$ has another interpretation as the dislocation density function at the critical time $t_{\mathrm{cr}}$.

Substituting Eqs. (16) and (10) into Eq. (13), one obtains the governing equation for the flux of incremental dislocations:

$$
\gamma\left[\int_{\eta}^{1} B_{i}(s) \mathrm{d} s\right]=\frac{1}{\sqrt{2 \pi x_{1}}}\left\{\frac{K_{\mathrm{M}}}{\mu_{i}}-\frac{b}{\sqrt{2 \pi l_{\mathrm{cr}}}} F_{i}^{+}\left(\frac{1}{v}\right) \int_{\eta_{i}}^{1} F_{i}^{-}\left(\frac{s}{v \eta}\right) \frac{\sqrt{s} B_{i}(s)}{s-\eta} \mathrm{d} s\right\} .
$$

The governing equation (17) is valid for $x_{1}$ ranging from 0 to $l_{\mathrm{cr}}$ (or for $\eta$ ranging from 0 to 1 ), with $l_{\text {cr }}$ furnished by Eq. (12). It is a singular nonlinear integral equation and casts in the form of generalized Peierls equation. Numerical solution for the dislocation density $B_{i}(s)$ and the slip length $l_{\text {cr }}$ can be facilitated by a Chebyshev polynomial expansion to remove the singular integration in Eq. (17). Coefficients of the expansion are solved by Newton-Raphson iteration. Details of the numerical scheme are illustrated in the appendix, as an extension for the scheme used by Beltz and Rice (1991, 1992), by Rice and Beltz (1994), and by Wang et al. (1996). When $B_{i}(s)$ is known, one can integrate according to Eq. (16) to get the displacement jump $\delta_{i}$ at the critical time.

Alternatively, one may change the dislocation density function $B_{i}$ to the inter-planar slip $\delta_{i}$, then Eq. (17) is formulated in a different form:

$$
\begin{aligned}
& \gamma\left[\frac{\delta_{i}\left(x_{1}, l_{\mathrm{cr}}\right)}{b}\right] \\
& =\frac{1}{\sqrt{2 \pi x_{1}}}\left\{\frac{K_{\mathrm{M}}}{\mu_{i}}+\frac{F_{i}^{+}(1 / v)}{\sqrt{2 \pi}} \int_{0}^{l_{\mathrm{cr}}-\left(v / c_{i}\right) x_{1}} \frac{\sqrt{l}}{l-x_{1}} F_{i}^{-}\left(\frac{l}{v x_{1}}\right) \frac{\mathrm{d} \delta_{i}(l)}{\mathrm{d} l} \mathrm{~d} l\right\} .
\end{aligned}
$$

In the quasi-static limit, the above formulation naturally reduces to the one proposed by Rice (1992),

$$
\begin{aligned}
& \gamma\left[\frac{\delta_{i}\left(x_{1}, l_{\mathrm{cr}}\right)}{b}\right]=\frac{1}{2 \pi \sqrt{x_{1}}} \int_{0}^{l_{\mathrm{cr}}}\left(\frac{\sqrt{l}}{l-x_{1}}-\frac{1}{\sqrt{l}}\right) \frac{\mathrm{d} \delta_{i}(l)}{\mathrm{d} l} \mathrm{~d} l, \\
& \int_{0}^{l_{\mathrm{cr}}} \frac{\mathrm{d} \delta_{i}(l)}{\mathrm{d} l} \frac{\mathrm{d} l}{\sqrt{l}}=-\sqrt{2 \pi} \frac{K_{\mathrm{M}}}{\mu_{i}} .
\end{aligned}
$$




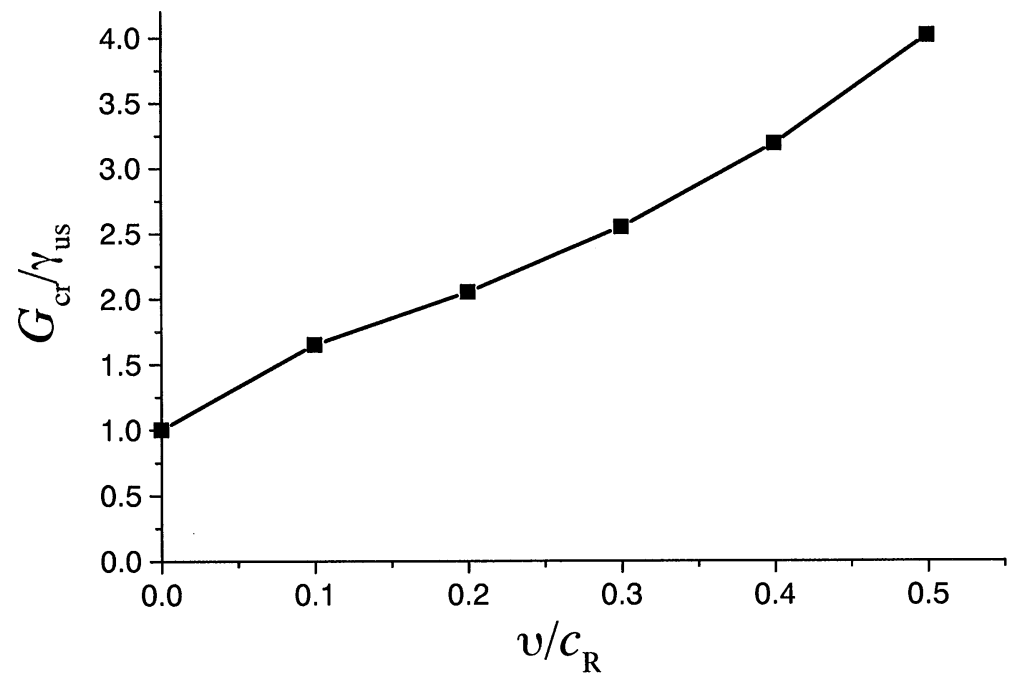

Fig. 2. Critical energy release rates $G_{\mathrm{cr}} / \gamma_{\mathrm{us}}$ for spontaneous nucleation of a transient dislocation of normalized velocity $v / c_{\mathrm{R}}$ for mode II.

Under the periodic relation (14), the solution of Eq. (17) is non-unique. Under the same load, the displacement jump at the crack tip $\delta_{i}\left(0, l_{\mathrm{cr}}\right)$ may locate at different intervals of $\tau$ versus $\delta$ curve. If $\delta_{i}\left(0, l_{\text {cr }}\right)$ locates at the ascending portion of the $\tau-\delta$ curve, the solution is stable while the solution is unstable if $\delta_{i}\left(0, l_{\mathrm{cr}}\right)$ locates at the descending portion. The remote load can be expressed by the energy release rate. Since the crack is non-propagating, the energy release rate relates to the applied stress intensity factors by

$$
G=\frac{1-v^{2}}{E}\left(K_{\mathrm{I}}^{2}+K_{\mathrm{II}}^{2}\right)+\frac{1}{2 \mu} K_{\mathrm{III}}^{2}
$$

Under prescribed $G$, a moving dislocation will nucleate spontaneously if $\delta_{i}\left(0, l_{\text {cr }}\right)$ exceeds a pivotal value of $b / 2$. Otherwise the transient dislocation has to nucleate by thermal activation. The critical energy release rate that causes a crack tip displacement jump of $b / 2$ is labeled by $G_{\mathrm{cr}}$. When the remote energy release rate reaches $G_{\mathrm{cr}}$, a complete dislocation may be nucleated and is ready to emit. Fig. 2 plots the mode II curve of the required applied load levels $G_{\mathrm{cr}} / \gamma_{\mathrm{us}}$ versus the normalized dislocation velocity $v / c_{R}$. The critical energy release rate increases as the dislocation velocity increases. The calculation was carried out under a fixed set of materials parameters. The Poisson's ratio $v$ is taken as 0.3 , and the Rayleigh wave speed is fixed at $c_{R}=$ $2000 \mathrm{~m} / \mathrm{s}$. Relations among the longitudinal, transverse and Rayleigh wave speeds (see, for example, Freund, 1990) lead to $c_{\mathrm{L}}=4040 \mathrm{~m} / \mathrm{s}$ and $c_{\mathrm{T}}=2159 \mathrm{~m} / \mathrm{s}$.

The discussion in the sequel is restricted to the case of $G \leqslant G_{\mathrm{cr}}$. Fig. 3 plots typical $\delta / b$ versus $x_{1} / b$ curves under different applied load levels $G / \gamma_{\text {us }}$ for mode II case. The solution in the first ascending portion of the $\tau-\delta$ curve is termed the first stable solution, and plotted in solid lines. The first stable solution shares the feature 

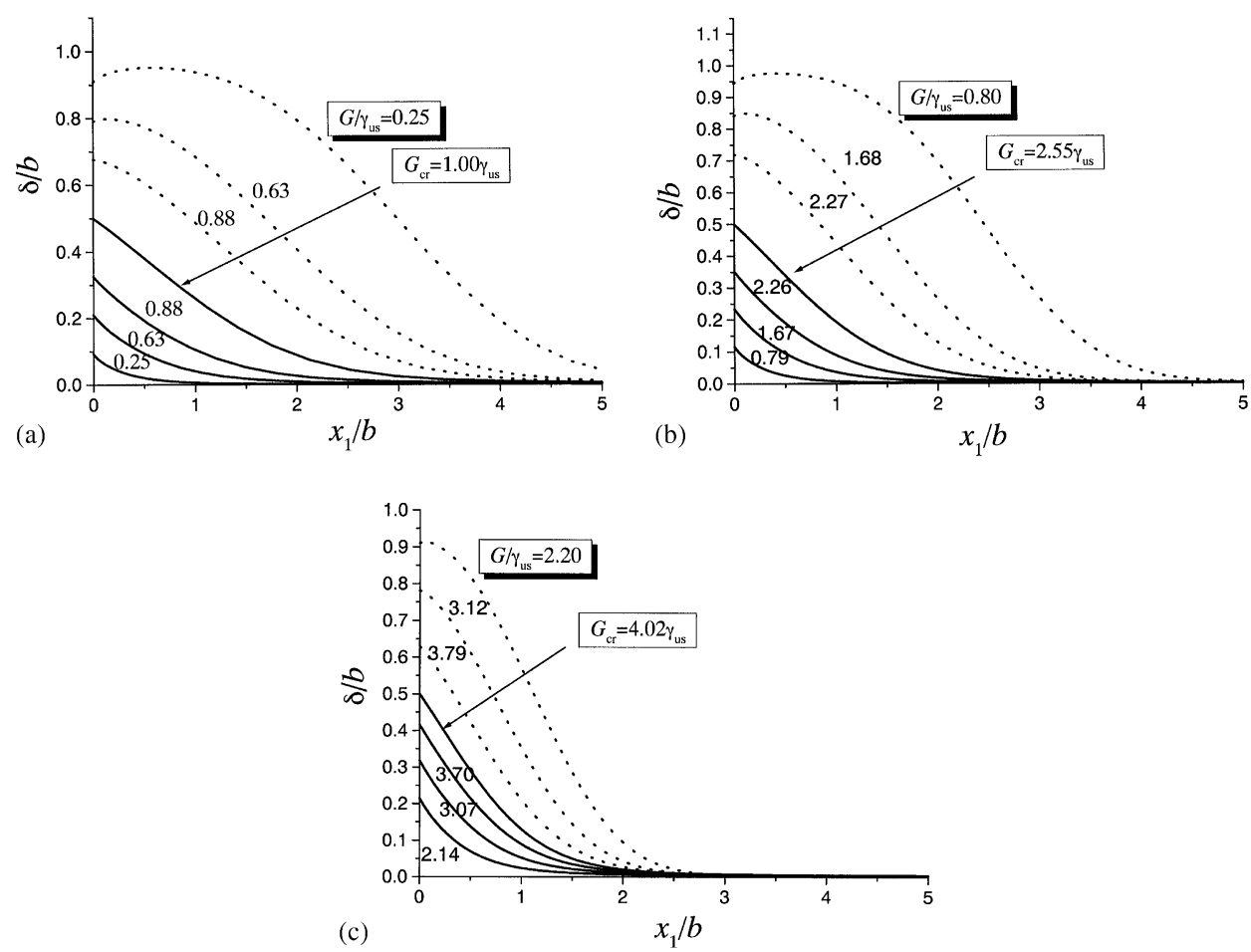

Fig. 3. $\delta / b$ versus $x_{1} / b$ curves for transient Peierls-Nabarro dislocation under different applied load, mode II: (a) $v=0$; (b) $v=0.3 c_{\mathrm{R}}$; (c) $v=0.5 c_{\mathrm{R}}$.

that the displacement jump along the crack extension line decreases rapidly from its maximum value at the crack tip to zero at $x_{1}=l_{\mathrm{cr}}$. The solution in the first descending portion of the $\tau-\delta$ curve is termed the first unstable solution and plotted in dot lines. These curves might climb up somewhat and then decline. Graph (a) corresponds to the quasi-static limit. The result from the present calculation agrees with that reported by Rice and Beltz (1994), giving confirmation for the present numerical scheme. Graphs (b) and (c) correspond to the case of $v=0.3 c_{\mathrm{R}}$ and $0.5 c_{\mathrm{R}}$, respectively. As predicted in Fig. 2, the load levels to produce similar displacement jump increase as the dislocation emission speed $v$ increases. Under the same applied load, the excited displacement discontinuity is smaller and more focused near the crack tip for the transient nucleation than those for the quasi-static dislocation nucleation.

When $G<G_{\mathrm{cr}}$, the dislocation has to be nucleated by thermal activation. The energy required for the thermal activation depends on the traveling speed of nucleated dislocation, while the latter has to exceed an escape velocity (a concept to be introduced in the next section) to accomplish dislocation emission. Transient dislocation nucleation excites both the strain energy and the kinetic energy inside the solid. The kinetic energy radiates out from the crack tip and the moving dislocation, and complicates the direct link between the activation energy and the inter-planar lattice potential. However, the 


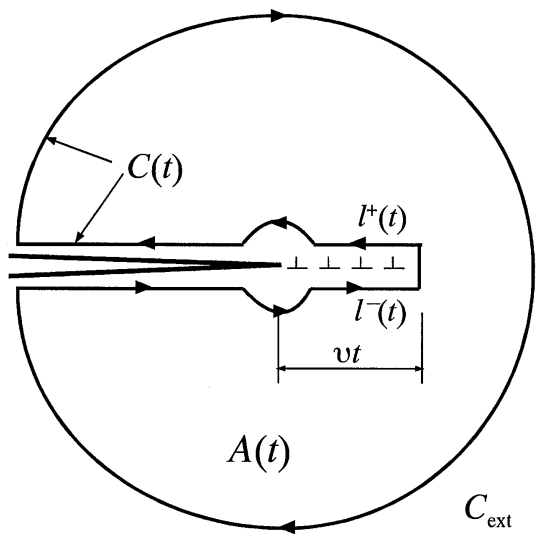

Fig. 4. Configuration to evaluate the differential energy before and after the nucleation of a transient dislocation. Area $A(t)$ is bounded by contour $C(t)$ composed of four parts: the exterior curve $C_{\text {ext }}$, the crack faces, a small circle around the crack tip, and the horizontal paths $l^{+}(t)$ and $l^{-}(t)$.

difference in energy (strain energy plus kinetic energy) before and after the nucleation of a transient dislocation can be evaluated by a time evolving contour shown in Fig. 4 . Through integration of the linear momentum equation in the domain of space and time, Freund (1990, Section 5.2) stated an energetic relation among the energies evaluated at different times and the stress power accumulated through its boundary. Applying his result to the present case of a stationary crack with a discontinuity strip extending with time, we have

$$
\begin{aligned}
\Delta U\left[\delta_{i}\left(x_{1}, t_{\mathrm{cr}}\right)\right]= & U_{\text {interplanar }}\left[\delta_{i}\left(x_{1}, t_{\mathrm{cr}}\right)\right]+\iint_{A\left(t_{\mathrm{cr}}\right)}(W+T) \mathrm{d} A \\
& -\iint_{A(0)}(W+T) \mathrm{d} A \\
= & \int_{0}^{l_{\mathrm{cr}}} \Phi\left[\delta_{i}\left(x_{1}, t_{\mathrm{cr}}\right)\right] \mathrm{d} x_{1}+\int_{0}^{t_{\mathrm{cr}}} \oint_{C(t)} \sum_{i} \sum_{j} \sigma_{j i} n_{j} \frac{\partial u_{i}}{\partial t} \mathrm{~d} C \mathrm{~d} t .
\end{aligned}
$$

In Eq. (21), $U_{\text {interplanar }}\left[\delta_{i}\left(x_{1}, t_{\mathrm{cr}}\right)\right]$ denotes the inter-planar energy due to a displacement jump of $\delta_{i}\left(x_{1}, t_{\mathrm{cr}}\right)$ caused by the incipient dislocation, and the symbols $W$ and $T$ denote the strain energy and the kinetic energy within the area of $A(t)$, bounded by the curve $C(t)$ shown in Fig. 4. The first equality of Eq. (21) states that the total energy gained by nucleating an incipient dislocation (denoted by $\Delta U$ ) equals the energy stored in the inter-planar bonds by forming a Peierls-Nabarro dislocation, plus the strain energy and the kinetic energy changes within the continuum. The second equality of Eq. (21) consists of two manipulations. First, the inter-planar energy is evaluated by the inter-planar potential $\Phi\left[\delta_{i}\left(x_{1}, t_{\mathrm{cr}}\right)\right]$, as discussed in several works (Schoeck, 1991; Beltz and Rice, 1991, 1992; Rice, 1992; Rice et al., 1992; Rice and Beltz, 1994), while 
the kinetic energy of inter-planar bonding is ignored due to its negligible inertia. The other manipulation converts the energy change within the continuum by the energy flux accumulated along the evolving contour $C(t)$ with an outside unit normal $n_{i}$. We divide $C(t)$ into four parts: the exterior curve $C_{\text {ext }}$, the crack faces, a small circle enclosing the crack tip, and the horizontal paths $l^{+}(t)$ and $l^{-}(t)$ above and below the incipient Peierls-Nabarro dislocation. Along the exterior curve $C_{\text {ext }}$, traction is prescribed and is independent of time. Prior to the arrival of stress wave generated by the transient dislocation, there is no energy flow through $C_{\text {ext }}$. Consequently, there is no contribution to the energy flux integral if $C_{\text {ext }}$ is drawn sufficiently far from the crack tip. The traction-free condition along the crack face eliminates its contribution to the energy equation. Moreover, the contribution from a circle around the crack tip vanishes as the circle shrinks onto the crack tip, since the particle velocity field $\mathrm{d} u_{i} / \mathrm{d} t$ is non-singular near a stationary crack tip. The only contribution of the last term in Eq. (21) comes from the discontinuity line created by the incipient dislocation. Above and beneath the incipient dislocation, the normals are given by $\left(n_{1}, n_{2}\right)=(0, \mp 1)$ and non-trivial displacements are $u_{i}^{ \pm}$in the path of $l^{ \pm}(t)$. Recall that $\delta_{i}=u_{i}^{+}-u_{i}^{-}$, one reduces Eq. (21) to

$$
\Delta U\left[\delta_{i}\left(x_{1}, t_{\mathrm{cr}}\right)\right]=\int_{0}^{l_{\mathrm{cr}}} \Phi\left[\delta_{i}\left(x_{1}, t_{\mathrm{cr}}\right)\right] \mathrm{d} x_{1}-\int_{0}^{t_{\mathrm{cr}}} \int_{0}^{v t} \sigma_{2 i}\left(x_{1}, t\right) \frac{\partial \delta_{i}\left(x_{1}, t\right)}{\partial t} \mathrm{~d} x_{1} \mathrm{~d} t .
$$

Substituting the expression (9) for $\delta_{i}$ into Eq. (22), and switching the order of integration, one obtains

$$
\begin{aligned}
& \int_{0}^{t_{\mathrm{cr}}} \int_{0}^{v t} \sigma_{2 i}\left(x_{1}, t\right) \frac{\partial \delta_{i}\left(x_{1}, t\right)}{\partial t} \mathrm{~d} x_{1} \mathrm{~d} t \\
& \quad=b l_{\mathrm{cr}} \int_{0}^{1} B_{i}\left(s^{*}\right)\left\{\int_{1-s^{*}}^{1} \sigma_{2 i}\left[l_{\mathrm{cr}}\left(s+s^{*}-1\right), t_{\mathrm{cr}} s\right] \mathrm{d} s\right\} \mathrm{d} s^{*} .
\end{aligned}
$$

Substituting Eq. (10) for $\sigma_{2 i}$ into Eq. (23), changing the order of integration, then substituting back into Eq. (22), one finally arrives at

$$
\begin{aligned}
\Delta U\left[\delta_{i}\left(x_{1}, t_{\mathrm{cr}}\right)\right]= & \int_{0}^{l_{\mathrm{cr}}} \Phi\left[\delta_{i}\left(x_{1}, t_{\mathrm{cr}}\right)\right] \mathrm{d} x_{1}-K_{\mathrm{M}} \int_{0}^{l_{\mathrm{cr}}} \frac{\delta_{i}\left(x_{1}, t\right)}{\sqrt{2 \pi x_{1}}} \mathrm{~d} x_{1} \\
& +\frac{\mu_{i} b^{2}}{2 \pi} F_{i}^{+}\left(\frac{1}{v}\right) \int_{0}^{1} B_{i}\left(s^{*}\right) \int_{v / c_{i} s^{*}}^{1} \Omega_{i}\left(\frac{s^{*}}{s} ; \frac{c_{i}}{v}\right) B_{i}\left(s^{\prime}\right) \mathrm{d} s^{\prime} \mathrm{d} s^{*}
\end{aligned}
$$

where

$$
\Omega_{i}(r ; q)= \begin{cases}\int_{0}^{r} F_{i}^{-}\left(\frac{1}{v \rho}\right) \frac{\mathrm{d} \rho}{\sqrt{\rho}(1-\rho)^{2}} & \text { if } r<1, \\ -\int_{r}^{q} F_{i}^{-}\left(\frac{1}{v \rho}\right) \frac{\mathrm{d} \rho}{\sqrt{\rho}(1-\rho)^{2}} & \text { if } r>1 .\end{cases}
$$

In the spirit of Rice and Beltz (1994), the activation energy for an incipient dislocation moving at a velocity $v$ may be defined as the energy barrier between two possible solutions of Eqs. (17) and (12). The activation energy for the nucleation of a straight 


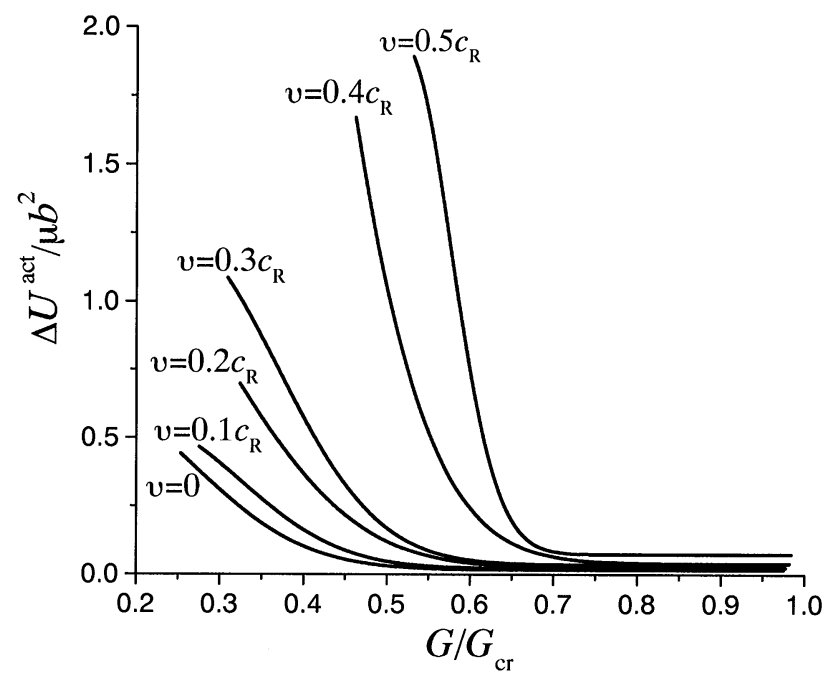

Fig. 5. Two-dimensional normalized activation energy $\Delta U^{\text {act }} /\left(\mu b^{2}\right)$ versus the normalized energy release rate $G / G_{\mathrm{cr}}$, under the dislocation velocity $v / c_{\mathrm{R}}=0,0.1,0.2,0.3,0.4,0.5$.

dislocation is

$$
\Delta U^{\mathrm{act}}\left[\delta_{i}\left(x_{1}, t_{\mathrm{cr}}\right)\right]=\Delta U\left[\delta_{i}^{\mathrm{sad}}\left(x_{1}, t_{\mathrm{cr}}\right)\right]-\Delta U\left[\delta_{i}^{\min }\left(x_{1}, t_{\mathrm{cr}}\right)\right],
$$

where $\delta_{i}^{\min }\left(x_{1}, t_{\mathrm{cr}}\right)$ denotes the first stable solution, and $\delta_{i}^{\mathrm{sad}}\left(x_{1}, t_{\mathrm{cr}}\right)$ the first unstable solution. Fig. 5 shows the normalized activation energy $\Delta U^{\text {act }} /\left(\mu b^{2}\right)$ versus the applied loads $G / G_{\text {cr }}$ curve for mode II case, under $v / c_{\mathrm{R}}$ values of $0,0.1,0.2,0.3,0.4$ and 0.5 . At a fixed dislocation velocity, the energy required to activate a dislocation declines as the applied energy release rate increases, and diminishes when $G$ approaches $G_{\mathrm{cr}}$. At a fixed value of applied stress intensity factor, the activation energy for dislocation nucleation increases with the emitting velocity. The increased activation energy serves to inject more kinetic energy to nucleate a fast moving dislocation.

\section{Dislocation escape from a crack tip}

Assisted by the thermal activation process, a moving dislocation is formed under the Peierls-Nabarro model described in the previous section. The fate of such a nucleated dislocation raises the next question: can it escape from the crack tip? Wang et al. (1996) devised a Peierls-Nabarro approach that unifies the formulation for dislocation nucleation and dislocation escape in the quasi-static case. Mathematical intricacy for a transient analysis, however, makes a unified Peierls-Nabarro formulation difficult for transient dislocation emission. To simplify the discussion, we replace the PeierlsNabarro dislocation to a fully developed Volterra dislocation. The escape problem is formulated by a complete dislocation of magnitude $b$, which nucleates at $\xi=\xi_{0}$ and moves at a velocity $v_{0}$ under an applied stress intensity factor $K_{\mathrm{M}}$. The activation 
energy required to nucleate such a dislocation can be found from Fig. 5. The range of $\xi_{0}$ falls within $1-1.5 b$. The total force $f_{i}\left(\xi_{0}, v_{0}\right)$ on such a dislocation consists of the driving force by the remote $K$ field, the crack tip drag on the transient dislocation, and the lattice resistance to the dislocation motion. Accordingly,

$$
\begin{aligned}
f_{i}\left(\xi_{0}, v_{0}\right)= & b\left\{\frac{K_{\mathrm{M}}}{\sqrt{2 \pi \xi_{0}}}-\frac{\mu_{i} b}{2 \pi \xi_{0}} F_{i}^{+}\left(\frac{1}{v_{0}}\right)\right. \\
& {\left.\left[\frac{1}{2} F_{i}^{-}\left(\frac{1}{v_{0}}\right)+\frac{1}{v_{0}} \dot{F}_{i}^{-}\left(\frac{1}{v_{0}}\right)\right]-\tau_{\text {Lattice }}\left(v_{0}\right)\right\}, }
\end{aligned}
$$

where the second term in the curved parentheses is evaluated through Eq. (5), and the last term represents the lattice resistance to the dislocation motion, commonly known as the dislocation mobility curve, see Hirth and Lothe (1982, Section 7.7). For a dislocation moves at an infinitesimal speed, one has

$$
f_{i}\left(\xi_{0}, 0\right)=b\left\{\frac{K_{\mathrm{M}}}{\sqrt{2 \pi \xi_{0}}}-\frac{\mu_{\mathrm{M}} b}{4 \pi \xi_{0}}-\tau_{\text {Lattice }}(0)\right\} .
$$

If $f_{i}\left(\xi_{0}, 0\right)$ is non-negative, a dislocation nucleated at $\xi_{0}$ can escape from the crack, regardless its velocity. The remaining part of the paper deals with the case of negative $f_{i}\left(\xi_{0}, 0\right)$. The nucleated dislocation at any velocity will first decelerate, that will lead to two possibilities. For a slow moving dislocation, it decelerates, then stops, and eventually retreats to the crack tip. On the other hand, a fast moving dislocation may overshoot the dragging barrier and escape from the crack.

The stress analysis for a dislocation moving at non-uniform speed is hardly tractable, except for the mode III case in which the exact expressions were given by Brock and co-workers (Brock, 1989; Brock and Wu, 1990a, b). For a smooth deceleration, we approximate the stress field in the vicinity of the moving dislocation by the stress field as if the dislocation moved out from the crack tip at the current velocity to the current location. We term this approximation the "quasi-steady approximation" for the stress field in the vicinity of the moving dislocation. For the special case of screw dislocations, the dislocation drag force derived under quasi-steady approximation happens to coincide with the exact analysis of Brock and Wu (1990a) for a screw dislocation moving at non-uniform speed. We anticipate that the quasi-steady approximation holds for a climb and an edge dislocation. Under this approximation, a Newtonian type equation for dislocation motion is derived as follows:

$$
\begin{aligned}
& \tilde{m}_{i} \frac{\mathrm{d} v}{\mathrm{~d} t}= b\left\{\frac{K_{\mathrm{M}}}{\sqrt{2 \pi \xi}}-\frac{\mu_{i} b}{2 \pi \xi} F_{i}^{+}\left(\frac{1}{v}\right)\left[\frac{1}{2} F_{i}^{-}\left(\frac{1}{v}\right)+\frac{1}{v} \dot{F}_{i}^{-}\left(\frac{1}{v}\right)\right]\right. \\
&\left.-\operatorname{sgn}(v) \tau_{\text {Lattice }}(v)\right\}, \\
& \frac{\mathrm{d} \xi}{\mathrm{d} t}=v, \quad \xi(0)=\xi_{0}, \quad v(0)=v_{0} .
\end{aligned}
$$


For the case of small $v / c_{\mathrm{T}}$, the "effective mass" $\tilde{m}_{i}$ of a dislocation is given by

$$
\tilde{m}_{1}=\frac{1+\left(c_{\mathrm{T}} / c_{\mathrm{L}}\right)^{4}}{c_{\mathrm{T}}^{2}} \frac{W_{0}}{L}, \quad \tilde{m}_{2}=\left[3 \frac{1+\left(c_{\mathrm{T}} / c_{\mathrm{L}}\right)^{4}}{c_{\mathrm{T}}^{2}}-\frac{4}{c_{\mathrm{L}}^{2}}\right] \frac{W_{0}}{L}, \quad \tilde{m}_{3}=\frac{1}{c_{\mathrm{T}}^{2}} \frac{W_{0}}{L}
$$

and $W_{0} / L=\left(\mu b^{2} / 4 \pi\right) \ln R / \xi_{0}$ denotes the self-energy per unit length of a straight and stationary screw dislocation. Symbol $R$ denotes the outside radius of the dislocation in its strain energy evaluation. The above expressions of the effective mass for a screw, an edge and a climb dislocation were due to Frank (1949), and Weertman $(1961,1967)$ and for the case that $v / c_{\mathrm{T}}$ is small. We notice in Eq. (29) the lattice resistance switches sign when the dislocation motion is reversed.

The equation system (29) describes a two-dimensional autonomous dynamic system in the $\xi-v$ phase plane. The trajectories in the phase plane are uniquely determined. Typical portraits for the phase trajectories are illustrated in Fig. 6a for the mode II case and Fig. $6 \mathrm{~b}$ for the mode III case, with abscissa $\xi / b$ and ordinate $v / c_{\mathrm{T}}$. Rate independent dislocation mobility curve $\tau_{\text {lattice }}(v)=\tau_{\mathrm{c}}$ is adopted. The material parameters are fixed at $K_{\mathrm{M}} / \mu \sqrt{b}=0.19$ and $\tau_{\mathrm{c}} / \mu=0.01$. To evaluate $W_{0} / L$ in Eq. (30), we choose the ratio $R / \xi_{0}$ as 1000 . The critical point for the system locates at $\xi=\tilde{\xi}$ and $v=0$ in the phase plane, with $\tilde{\xi}$ given by

$$
\tilde{\xi}=\frac{K_{\mathrm{M}}^{2}}{8 \pi \tau_{\mathrm{c}}^{2}}\left(1-\sqrt{1-\frac{2 \mu_{i} b}{K_{\mathrm{M}}^{2}} \tau_{\mathrm{c}}}\right)^{2}
$$

The condition of $K_{\mathrm{M}}>\sqrt{2 \mu_{i} b \tau_{\mathrm{c}}}$ is implied in this equation. Fig. 7a and b plot the normalized critical point $\tilde{\xi} / b$ with respect to the applied stress intensity factors, normalized by $\mu \sqrt{b}$, for an edge and a screw dislocation. For both cases, the critical points draw close the nucleation position as the remote stress intensity factor increases.

Under a fixed value of $K_{\mathrm{M}}$ less than the critical value of spontaneous nucleation, the escape velocity $\tilde{v}$ bears the following meaning. If $v_{0}<\tilde{v}$, a dislocation that nucleates at $\xi=\xi_{0}$ can never reach $\tilde{\xi}$; if $v_{0}>\tilde{v}$, the same dislocation can overshoot through $\tilde{\xi}$, and accelerate to escape from the crack tip. Only dislocations nucleated at larger than the escape velocity can emanate from the crack tip, though more activation energy is required for their nucleation. The escape velocity $\tilde{v}$ can be resolved as follows. One rewrites Eq. (29) as

$$
\begin{aligned}
\frac{\mathrm{d} v}{\mathrm{~d} \xi}= & \frac{b}{\tilde{m}_{i} v}\left\{\frac{K_{\mathrm{M}}}{\sqrt{2 \pi \xi}}-\frac{\mu_{i} b}{2 \pi \xi} F_{i}^{+}\left(\frac{1}{v}\right)\left[\frac{1}{2} F_{i}^{-}\left(\frac{1}{v}\right)+\frac{1}{v} \dot{F}_{i}^{-}\left(\frac{1}{v}\right)\right]\right. \\
& \left.-\operatorname{sgn}(v) \tau_{\text {Lattice }}(v)\right\},
\end{aligned}
$$

which is a first-order nonlinear ordinary differential equation in the phase plane. Integrating it from the critical point $\xi=\tilde{\xi}$ and $v=0$ backward, one obtains the escape velocity $\tilde{v}$ as the $v$ value at $\xi=\xi_{0}$. We choose $\xi_{0}=1.25 b$ in the numerical calculations. Fig. $8 \mathrm{a}$ and $\mathrm{b}$ plot the normalized escape velocities $\tilde{v} / c_{\mathrm{T}}$ with respect to the applied stress intensity factors, normalized by $\mu \sqrt{b}$, for an edge and a screw dislocation. For both cases, the escape velocity declines as the remote stress intensity factor increases, and diminishes when the remote stress intensity factor approaches the critical value for 


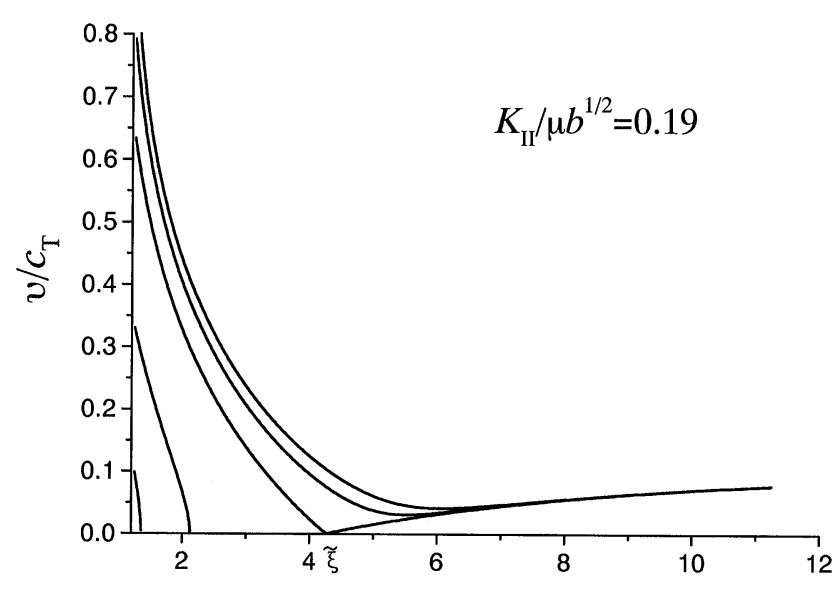

(a)

$\xi / b$

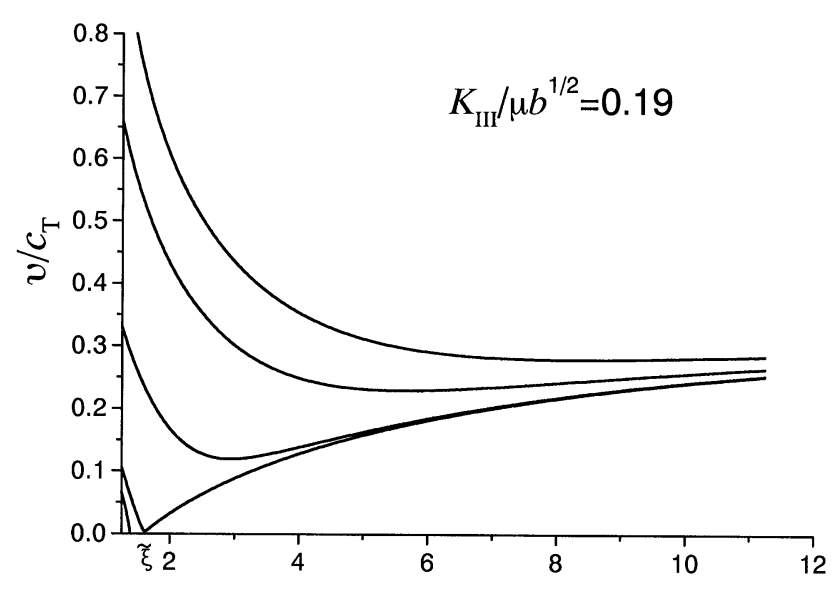

(b)

$\xi / b$

Fig. 6. Trajectories in the phase plan span by the abscissa $\xi / b$ and the ordinate $v / c_{\mathrm{T}}$, under a load level of $K_{\mathrm{M}} / \mu \sqrt{b}=0.19$ : (a) edge dislocation; (b) screw dislocation. Figs. 6 and 7 are plotted under $\tau_{\text {lattice }}(v)=\tau_{\mathrm{c}}=0.01 \mu$ and $R / \xi_{0}=1000$.

spontaneous nucleation. Combining Figs. 8 and 5, one concludes that the activation energy for a dislocation to escape from the crack tip decreases as the applied stress intensity factor increases.

\section{Loading rate effect on brittle-to-ductile transition}

Three-dimensional calculation for a protruding dislocation loop is cumbersome for the transient situation. For the quasi-static case, Rice and Beltz (1994) reported that 

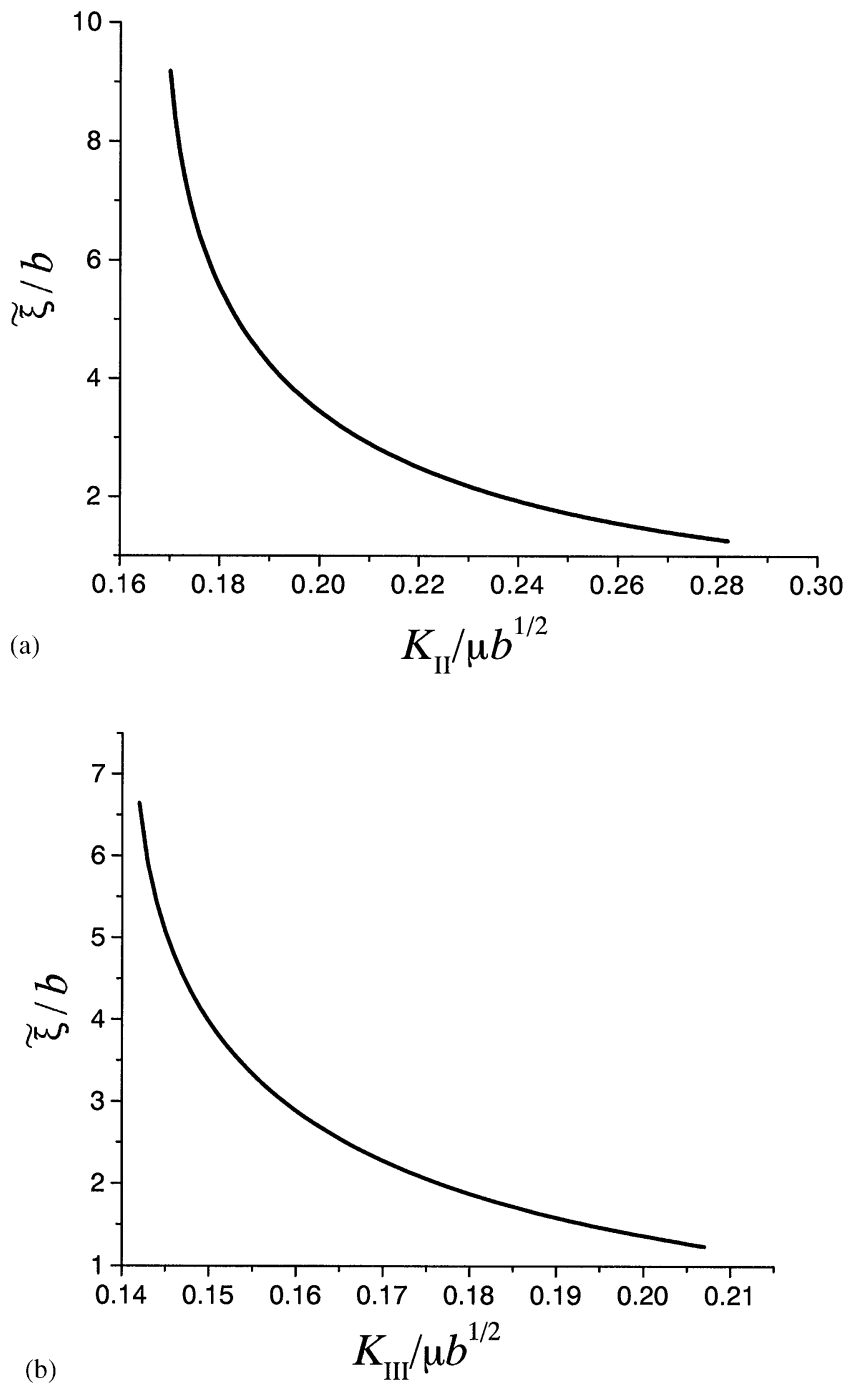

Fig. 7. Normalized critical point $\tilde{\xi} / b$ versus normalized applied stress intensity factor $K_{\mathrm{M}} /(\mu \sqrt{b})$ curves: (a) edge dislocation; (b) screw dislocation.

the three-dimensional activation energy $\Delta E$ for such a protruding loop approximately relates to the two-dimensional activation energy $\Delta U^{\text {act }}$ by

$$
\Delta E \approx 5 \sim 10 b \times \Delta U^{\text {act }} .
$$

For thermal activated nucleation, the nucleation rate is given by (Matthews et al., 1976)

$$
v_{\mathrm{nu}}=\frac{n_{\mathrm{nu}} c_{\mathrm{T}}}{b} \exp \left(-\frac{\Delta E}{K_{\mathrm{B}} \mathrm{T}}\right),
$$



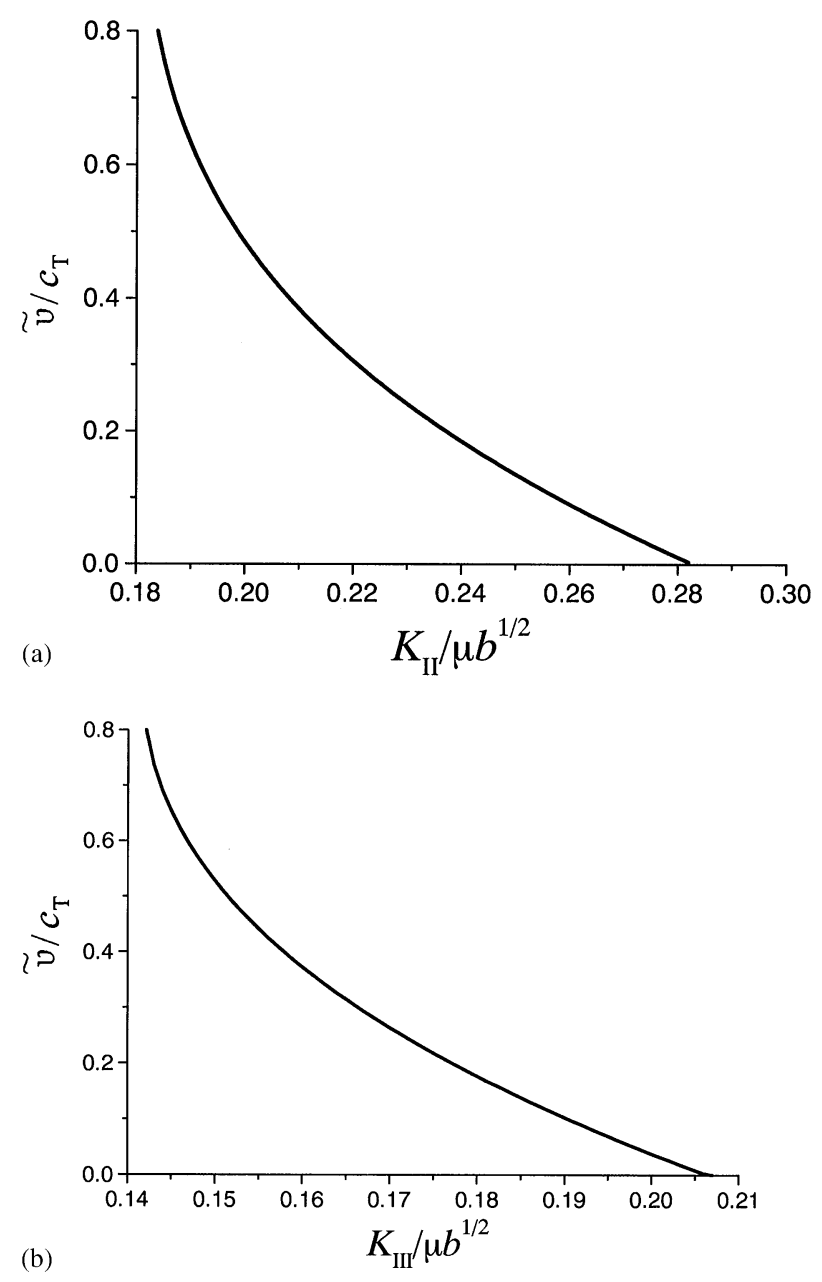

Fig. 8. Normalized escape velocity $\tilde{v} / c_{\mathrm{T}}$ versus normalized applied stress intensity factor $K_{\mathrm{M}} /(\mu \sqrt{b})$ curves, $\xi_{0}=1.25 b$ : (a) edge dislocation; (b) screw dislocation.

where $n_{\mathrm{nu}} \sim 1 /(10 b)$ is the number of nucleation sites per unit length of crack front, $K_{\mathrm{B}}$ the Boltzmann constant, and $T$ the temperature in Kelvin. Substituting Eqs. (33), (26) and (24) into Eq. (34), one finds that the nucleation rate decreases exponentially with respect to $v_{0}$. Recalling that $v_{0}$ has to exceed $\tilde{v}$ to escape from the crack tip, one realizes that the higher the escape velocity, the lower the nucleation rate of the dislocation.

As the loading rate varies, dislocation emission responds differently from cleavage. The load level to excite a transient dislocation depends on the loading rate; while the cleavage behavior, as a bond rupture process governed by a Griffith type energy criterion, depends only on the current stress along the cleavage plane near the crack tip, as demonstrated by many experiments, as well as by atomistic simulation (Tan 
and Yang, 1994a). Under a slow loading rate, there will be ample time for dislocation emission even if it requires a high level of activation energy to escape from the crack tip. Dislocation emission intervenes when the applied stress intensity factor $K_{\mathrm{M}}$ is considerably lower than the value for cleavage. Under a fast loading rate, the limited loading period only allows dislocations of low activation energy to escape from the crack tip. To lower the energy barrier, the applied energy release rate should be raised. Therefore, the load level at which transient dislocation emission occurs relies on the loading rate. Therefore, a fast loading rate promotes cleavage, or brittle material behavior; while a slow loading rate promotes dislocation emission, or ductile material behavior. The brittle to ductile transition of materials at a slower load rate is benefited from the available time to activate dislocations that overcome the energy barrier for a transient escape from the crack tip.

The following two cases may further quantify the discussion. First consider the case of $G_{\mathrm{cr}}(0)<G_{\text {cleave }}$. Prompt dislocation nucleation is achieved, and nucleated dislocations may eventually escape from the crack tip. Loading rate effect intervenes only if the dislocation emission is not fast enough to relieve the crack tip stress. To quantify the process, one can find an upper bound $v^{+}$of dislocation velocity from Fig. 2 according to the cleavage energy $G_{\text {cleave }}$ :

$$
G_{\mathrm{cr}}\left(v^{+}\right)=G_{\text {cleave }} \text {. }
$$

Dimensional consideration dictates that the maximum stress at the blunted crack tip scales with $\dot{K} \sqrt{b} / v^{+}$. A fast loading rate embrittles the material by demanding a high value of the dislocation emission speed $v^{+}$.

Next consider the case of $G_{\mathrm{cr}}(0)>G_{\text {cleave }}$. Thus dislocation nucleation controls the brittle-to-ductile transition. The rate of dislocation generation is given in Eq. (34) with the activation energy given by Eq. (33). That activation energy should be evaluated at a crack emission velocity whose lower bound is given by Fig. 8 as

$$
v^{-}=\tilde{v}\left(G_{\text {cleave }} / G_{\text {cr }}(0)\right) \text {. }
$$

On the other hand, the rate of stressing is measured by $\dot{K} / K_{\text {cleave }}$. Dimensional consideration dictates that the maximum stress at the blunted crack tip scales with $\dot{K} /\left(K_{\text {cleave }} v_{\text {nu }} b\right)$. At the brittle-to-ductile transition temperature $T_{\mathrm{c}}$, one should have

$$
\frac{1}{T_{\mathrm{c}}}=\frac{K_{\mathrm{B}}}{\Delta E\left(v^{-}\right)} \ln \left(\frac{C K_{\text {cleave }} n_{\mathrm{nu}} c_{\mathrm{T}}}{\dot{K}}\right),
$$

where $C$ is a dimensionless constant and the $\Delta E\left(v^{-}\right)$value is furnished by Eqs. (33), (36) and Fig. 3. The rate dependence of the transition temperature is supported by many testing data (Nitzsche and Hsia, 1994; Kim and Roberts, 1994; Hirsch and Roberts, 1996; Booth and Roberts, 1997; Ebrahimi and Shrivastava, 1998).

\section{Concluding remarks}

Transient emission of dislocation from a crack tip gives a new twist to the brittle-toductile behavior of the materials. Two competitions are involved: the competition between cleavage and dislocation emission, and the competition between transient 
nucleation and transient escape of dislocations from a crack tip. A message of this paper is the latter competition, along with its consequence that a brittle to ductile transition depends on the loading rate, as supported by many testing data. The transient nucleation of a dislocation requires extra activation energy; the faster the moving speed, the higher the level of energy to activate it. On the other hand, the momentum of a fast moving dislocation, when nucleated, helps it to overrun the dragging barrier by the crack tip attraction. Though at a slow nucleation rate, a fast moving dislocation can escape from the crack tip at a relatively low load level, while a slow moving dislocation, albeit attempted at rapid nucleation frequency, might fail to get away from the crack tip. This scenario leads to the loading rate dependency of brittle to ductile transition commonly observed in experiments.

Three assumptions are incorporated in the present analysis, as tradeoffs to explore this complicated phenomenon. First, we confine the plane of dislocation emission to the crack extension plane. That geometry precludes the possibility of crack tip blunting, as well as the possibility that cleavage and dislocation emission are presided by different stress distributions. Second, during the transient nucleation of a Peierls-Nabarro dislocation, the inter-planar relation and the crack tip shielding condition are enforced only at the moment when its nucleation possibility is examined. During the intermediate time period, the process is so transient that these two conditions cannot be maintained. Actually, they can be satisfied only if incremental dislocations travel at non-uniform speeds, and consequently a clear definition on the nucleation speed of the dislocation is lost. The third assumption is the quasi-steady approximation in the analysis for the dislocation escape process. This assumption is confirmed in the case of non-uniformly moving screw dislocations, in which the quasi-steady approximation delivers the exact result of dislocation drag force as obtained by Brock and Wu (1990a). Further research is needed to clarify those approximations.

\section{Acknowledgements}

W. Yang and J.C. Tang would like to thank Prof. G. Beltz of University of California, Santa Barbara, and Prof. T.-C. Wang of the Institute of Mechanics, Chinese Academy of Sciences, for sharing with us their numerical experience in dealing with the nonlinear singular integral equation for a Peierls-Nabarro dislocation.

\section{Appendix Numerical scheme to solve integral equation (17)}

In the spirit of Wang et al. (1996), the dislocation density function $B_{i}(s)$, when multiplied by $\sqrt{s}$, can be expanded in a truncated sinusoidal series:

$$
\sqrt{s} B_{i}(s)=\frac{1}{2} \sum_{n=1}^{N} \beta_{n} \sin n \theta \quad \text { with } s=\frac{1}{2}(1+\cos \theta) .
$$

In the numerical solution, $N$, the number of truncated terms, is chosen as 180 , a sufficiently large integer. The sinusoidal expansion (A.1) is equivalent to an expansion 
of Chebyshev polynomials of the second kind. Substituting Eq. (A.1) into Eq. (17), one obtains

$$
\gamma\left[\sum_{n=1}^{N} \beta_{n} D_{n}(\eta)\right]=\frac{1}{\sqrt{2 \pi l_{\mathrm{cr}} \eta}}\left\{\frac{K_{\mathrm{M}}}{\mu_{i}}-\frac{b}{\sqrt{2 \pi l_{\mathrm{cr}}}} F_{i}^{+}\left(\frac{1}{v}\right) \sum_{n=1}^{N} \beta_{n} T_{n}(\eta)\right\},
$$

where

$$
\begin{aligned}
D_{n}(\eta)= & \frac{\sin (n-1 / 2) \psi}{2(2 n-1)}-\frac{\sin (n+1 / 2) \psi}{2(2 n+1)} \\
T_{n}(\psi)= & -\frac{\pi}{2} F_{i}^{-}\left(\frac{1}{v}\right) \cos n \psi-\frac{1}{2} F_{i}^{-}\left(\frac{1}{v}\right) \int_{\psi_{i}}^{\pi} \frac{\sin n \theta \sin \theta}{\cos \theta-\cos \psi} \mathrm{d} \theta, \\
& +\frac{1}{2} \int_{0}^{\psi_{i}}\left\{F_{i}^{-}\left[\frac{1+\cos \theta}{1+\cos \psi} \frac{1}{v}\right]-F_{i}^{-}\left(\frac{1}{v}\right)\right\} \frac{\sin n \theta \sin \theta}{\cos \theta-\cos \psi} \mathrm{d} \theta
\end{aligned}
$$

and $\psi=\cos ^{-1}(2 \eta-1), \psi=\cos ^{-1}\left(2 \eta_{i}-1\right)$. In Eq. (A.4), the derivation of the first term involves the integration of a singular kernel by means of the property of Chebyshev polynomials, the integration of the second term is free of singularity for $v<c_{\mathrm{R}}$, while the singularity at $\theta=\psi$ is removed in the third term. Thus, the integration in Eq. (A.4) can be carried out by conventional numerical integration routines.

Eq. (A.2) can be discretized at $N$ points $\left(\eta_{1}, \ldots, \eta_{N}\right)$ :

$$
\eta_{m}=\left(1+\cos \theta_{m}\right) / 2, \quad \theta_{m}=\frac{m-1}{N} \pi, \quad m=1,2, \ldots, N .
$$

A condition complementary to this system is furnished by substituting Eq. (A.1) into Eq. (12):

$$
\sqrt{2 \pi l_{\mathrm{cr}}} K_{\mathrm{M}}=\frac{\pi}{2} \mu_{i} b F_{i}^{+}\left(\frac{1}{v}\right) \sum_{n=1}^{N}(-1)^{n+1} \beta_{n} .
$$

Eq. (A.6) provides a relation between $K_{\mathrm{M}}$ and $l_{\mathrm{cr}}$, either of them may serve as the loading parameter. If one selects $l_{\text {cr }}$ as the loading parameter, (see Wang, 1995), one can substitute Eqs. (13)-(15) and (A.6) into Eq. (A.2) to arrive at

$$
\frac{4 \pi \gamma_{\mathrm{us}} l_{\mathrm{cr}}}{\mu_{i} b^{2}} \sin \frac{2 \pi \Delta_{m}}{b}=\sum_{n=1}^{N} \frac{\beta_{n}}{\sqrt{\eta_{m}}}\left[\cos (n \pi)-\frac{2}{\pi} T_{n}\left(\eta_{m}\right)\right] .
$$

Eqs. (14), (16), (A.1) and $\Delta=\delta+\tau / \mu$ lead to a relation between $\beta_{n}$ and $\Delta_{m}$. For the first stable solution, the scheme of using $\beta_{n}$ as the basic variable gives converge result. The governing equation becomes

$$
\frac{4 \pi \gamma_{\mathrm{us}} l_{\mathrm{cr}}}{\mu_{i} b^{2}} \sin \left(2 \pi \sum_{n=1}^{N} B_{m n} \beta_{n}\right)=\sum_{n=1}^{N} A_{m n} \beta_{n},
$$


where

$$
A_{m n}=\frac{\cos (n \pi)-(2 / \pi) T_{n}\left(\theta_{m}\right)}{\sqrt{\eta_{m}}}, \quad B_{m n}=D_{n}\left(\theta_{m}\right)+\frac{b}{4(1-v) l_{\mathrm{cr}}} F_{i}^{+}\left(\frac{1}{v}\right) A_{m n} .
$$

For the first unstable solution, the scheme of using $\Delta_{m}$ as the basic variable gives converge result. The governing equation becomes:

$$
\begin{aligned}
\Delta_{m}= & \frac{\pi \gamma_{\mathrm{us}}}{\mu}\left\{\frac{4(1-v) l_{\mathrm{cr}}}{b} \sum_{n=1}^{N} \sum_{k=1}^{N} A_{n k}^{-1} \sin \left(\frac{2 \pi \Delta_{k}}{b}\right) D_{n}\left(\eta_{m}\right)\right. \\
& \left.+F_{i}\left(\frac{1}{v}\right) \sin \left(\frac{2 \pi \Delta_{m}}{b}\right)\right\} .
\end{aligned}
$$

The solution can be carried out by Newton-Raphson method.

\section{References}

Argon, A.S., 1987. Brittle to ductile transition in cleavage fracture. Acta Metall. 35, 185-196.

Beltz, G.E., Rice, J.R., 1991. Dislocation nucleation versus cleavage decohesion at crack tips. In: Lowe, T.C., Rollett, A.D., Follansbee, P.S., Daehn, G.S. (Eds.), Modeling the Deformation of Crystalline Solids: Physical Theory, Application, and Experimental Comparisons. TMS Minerals, Metals and Materials Society, Warrendale, PA, pp. 457-480.

Beltz, G.E., Rice, J.R., 1992. Dislocation nucleation at metal/ceramic interfaces. Acta Metall. 40, S321-S331.

Booth, A.S., Roberts, S.G., 1997. The brittle-ductile transition in $\gamma$-TiAl single crystals. Acta Materialia 45, 1045-1053.

Brock, L.M., 1989. An exact transient analysis of dislocation emission and fracture. J. Mech. Phys. Solids 37, 47-69.

Brock, L.M., Jolles, M., 1987. Dislocation-crack edge interaction in dynamic brittle fracture and crack propagation. Int. J. Solids Struct. 23, 607-619.

Brock, L.M., Wu, J.-S., 1990a. An equation of motion for the emission of accelerating dislocations from a crack. J. Mech. Phys. Solids 38, 273-285.

Brock, L.M., Wu, J.-S., 1990b. Incident wave and dislocation acceleration effects in dislocation emission from cracks. ASME J. Appl. Mech. 57, 870-876.

Chiao, Y.-H., Clark, D.R., 1989. Direct observation of dislocation emission from crack tips in silicon at high temperatures. Acta Metall. 37, 203-217.

Ebrahimi, F., Shrivastava, S., 1998. Brittle-to-ductile transition in NiAl single crystal. Acta Materialia 46, 1493-1502.

Frank, F.C., 1949. On the equation of motion of crystal dislocations. Proc. Phys. Soc. 62A, 131-134.

Freund, L.B., 1974. The stress intensity factor due to normal impact loading of the faces of a crack. Int. J. Eng. Sci. 12, 179-189.

Freund, L.B., 1990. Dynamic Fracture Mechanics. Cambridge University Press, Cambridge.

Hirsch, P.B., Roberts, S.G., 1996. Comment on the brittle-to-ductile transition: a cooperative dislocation generation instability; dislocation dynamics and the strain-rate dependence of the transition temperature. Acta Materialia 44, 2361-2371.

Hirth, J.P., Lothe, J., 1982. Theory of Dislocations, 2nd Edition. Wiley, New York.

Hsia, K.J., Argon, A.S., 1994. Experimental study of the mechanisms of the brittle-to-ductile transition of cleavage fracture in Si single crystals. Mater. Sci. Eng. A176, 111-119.

Hsia, K.J., Suo, Z., Yang, W., 1994. Cleavage due to dislocation confinement in layered materials. J. Mech. Phys. Solids 42, 877-896.

Kelly, A., Tyson, W.R., Cottrell, A.H., 1967. Ductile and brittle crystals. Phil. Mag. 15, 567-586. 
Kim, H.S., Roberts, S.G., 1994. Brittle-ductile transition and dislocation mobility in sapphire. J. Amer. Ceramic Soc. 77, 3099-3104.

Kobayashi, S., Ohr, S.M., 1980. In situ fracture experiments in b.c.c. metals. Philos. Mag. A 42, $763-772$.

Kobayashi, S., Ohr, S.M., 1981. In situ observation of the formation of plastic zone ahead of a crack tip in copper. Scr. Metall. 15, 343-348.

Kobayashi, S., Ohr, S.M., 1984. Dislocation arrangement in the plastic zone of propagating cracks in nickel. J. Mater. Sci. 19, 2273-2277.

Matthews, J.W., Blakeslee, A.E., Mader, S., 1976. Use of misfit strain to remove dislocations from epitaxial thin films. Thin Soild Films 33, 253-266.

Nitzsche, V.R., Hsia, K.J., 1994. Modelling of dislocation mobility controlled brittle-to-ductile transition. Mater. Sci. Eng. A176, 155-164.

Ohr, S.M., 1985. An electron microscope study of crack tip deformation and its impact on dislocation theory of fracture. Mater. Sci. Eng. 72, 1-35.

Ohr, S.M., 1988. HVEM observation of dislocation-free zones at crack tips in iron single crystals. Philos. Mag. A 57, 677-684.

Rice, J.R., 1992. Dislocation nucleation from a crack tip: an analysis based on the Peierls concept. J. Mech. Phys. Solids 40, 239-271.

Rice, J.R., Beltz, G.E., 1994. The activation energy for dislocation nucleation at a crack. J. Mech. Phys. Solids 42, 333-360.

Rice, J.R., Beltz, G.E., Sun, Y., 1992. Peierls framework for dislocation nucleation from a crack tip. In: Argon, A.S. (Ed.), Topics in Fracture and Fatigue Springer, Berlin, pp. 1-58 (Chapter 1).

Rice, J.R., Thomson, R., 1974. Ductile versus brittle behavior of crystals. Phil. Mag. 29, $73-97$.

Schoeck, G., 1991. Dislocation emission from crack tips. Phil. Mag. A 63, 111-120.

Schoeck, G., 1994. The generalized Peierls Nabarro model. Phil. Mag. A 69, 1085-1095.

Schoeck, G., 1996. Dislocation emission from crack tips as a variational problem of the crack energy. J. Mech. Phys. Solids 44, 413-437.

Tan, H.L., Yang, W., 1994a. Atomistic/continuum simulation of interfacial fracture, Part I: atomistic simulation. Acta Mech. Sinica 10, 151-162.

Tan, H.L., Yang, W., 1994b. Atomistic/continuum simulation of interfacial fracture, Part II: Combined atomistic/dislocation/continuum simulation. Acta Mech. Sinica 10, 237-249.

Tan, H.L., Yang, W., 1995. Nonlinear motion of atoms at a crack tip during a dislocation emission processes. J. Appl. Phys. 78, 7025-7034.

Wang, T.C., 1995. Dislocation nucleation and emission from crack tip. Int. J. Fracture 69, 295-306.

Wang, T.C., Wang, K.R., Zhang, Y.W., 1996. A unified model for dislocation nucleation, dislocation emission and dislocation free zone. Int. J. Fracture 78, 227-239.

Weertman, J., 1961. High velocity dislocations. In: Shewmon, G., Zackay, V.F. (Eds.), Response of Metals to High Velocity Deformation. Interscience, New York, pp. 205-247.

Weertman, J., 1967. Stress and displacement fields of an edge dislocation that climbs with a uniform velocity. J. Appl. Phys. 38, 2612-2614.

Xing, Y., Dai, F., Yang, W., 2000. Experimental study about nano-deformation field near quasi-cleavage crack tip. Sci. China 43, 963-968.

Yang, W., Tan, H.L., Guo, T.F., 1994. Evolution of crack tip process zone. Model Simul. Mater. Sci. Eng. 2, 767-782.

Zhu, T., Yang, W., Guo, T.F., 1996. Quasi-cleavage processes driven by dislocation pileups. Acta Metall. Mater. 44, 3049-3058. 